
limitation: pathophysiologic mechanisms. Am J Respir Crit Care Med 1997; 155: 109-115.

9 Gosselink R, De Vos J, van den Heuvel SP, et al. Impact of inspiratory muscle training in patients with COPD: what is the evidence? Eur Respir J 2011; 37: 416-425.

10 Wanke T, Formanek D, Lahrmann H, et al. Effects of combined inspiratory muscle and cycle ergometer training on exercise performance in patients with COPD. Eur Respir J 1994; 7: 2205-2211.

11 Langer D, Charususin N, Jacome C, et al. Efficacy of a novel method for inspiratory muscle training in people with chronic obstructive pulmonary disease. Phys Ther 2015; 95: 1264-1273.

12 Petrovic M, Reiter M, Zipko $\mathrm{H}$, et al. Effects of inspiratory muscle training on dynamic hyperinflation in patients with COPD. Int J Chron Obstruct Pulmon Dis 2012; 7: 797-805.

13 Collins EG, Langbein WE, Fehr L, et al. Can ventilation-feedback training augment exercise tolerance in patients with chronic obstructive pulmonary disease? Am J Respir Crit Care Med 2008; 177: 844-852.

14 Tzelepis GE, Vega DL, Cohen ME, et al. Lung volume specificity of inspiratory muscle training. J Appl Physiol 1994; 77: 789-794.

15 Tzelepis GE, Vega DL, Cohen ME, et al. Pressure-flow specificity of inspiratory muscle training. J Appl Physiol 1994; 77: 795-801.

\title{
Assessing small airway impairment in mild-to-moderate smoking asthmatic patients
}

\section{To the Editor:}

Asthma is characterised by airway inflammation throughout the bronchial tree, including the small airways. In asthma, small airway alterations are associated with poor clinical outcomes [1]. Cigarette smoke is known to induce peripheral airway abnormalities, asthmatic smokers exhibit a more rapid lung function decline, experience more frequent exacerbations and are more likely to be uncontrolled, even when the disease is managed as recommended [2]. The mechanisms accounting for poor responses to treatment in smoking asthmatic patients are currently unclear, although low accessibility to inhaled medications in the peripheral airways is considered a limiting factor for the efficacy of such treatments [3].

Currently, small-particle-size inhaled treatments (mass median aerodynamic diameter $<2 \mu \mathrm{m}$ ), which feature increased peripheral deposition relative to standard formulations, are available [4]. A recent observational study reported the efficacy of inhaled small-particle-size formulations in comparison to standard formulations, with regard to the improvements in clinical outcomes in a population of smoking asthmatic patients [5]. Thereby, suggesting that targeting peripheral alterations might yield clinical benefits in this population.

In this study, we hypothesised that cigarette smoking aggravates small airway abnormalities in asthmatic patients. The primary study outcome was an assessment of small airway dysfunction in smoking asthmatics. In addition, we evaluated the effect of switching the pre-existing standard-size particle inhaled treatment to an equipotent small-particle-size inhaled formulation.

Cross-sectional phase and primary outcome. Stable asthmatic patients (i.e. no exacerbations and changes in inhaled treatments during the 3 months preceding the study) were consecutively screened at the Respiratory Department of the University of Ferrara (Ferrara, Italy). Never smokers or current smokers (10-20 pack-years) with mild-to-moderate asthma who ranged in age from 18 to 50 years, free from an exacerbation for a minimum of 2 months and treated for at least 3 months with low-dose standard-particle-size inhaled corticosteroids (ICS) (step 2) alone or together with long-acting $\beta_{2}$ agonists (LABA) (ICS/LABA, fixed-dose combination; step 3) [6] were included. We adopted a lower cut-off limit of 10 pack-years, a usual standard in clinical trials, to qualify smokers. The upper limit of 20 pack-years was arbitrarily chosen to reduce the inclusion of subjects with advanced/irreversible chronic obstructive pulmonary disease-like peripheral alterations. Patients were excluded if they had been treated with small-particle-size formulations before entering the study, had irreversible airflow limitation after inhaling $400 \mu \mathrm{g}$ of albuterol (i.e. post bronchodilator forced expiratory volume in $1 \mathrm{~s}$ (FEV1)/forced vital capacity (FVC) $<70 \%$ ), or had an impaired diffusion capacity (predicted diffusing capacity of the lungs for carbon monoxide $<80 \%$ ). 
Longitudinal phase. After the initial assessment, patients entered a 3-month open-label treatment intervention. During this period, inhaled standard-particle-size treatments were switched to small-particle-size formulations at ICS equipotent doses, these included either: 1) small-particle-size beclomethasone (BDP) (Chiesi Farmaceutici Spa, Parma, Italy) alone for patients previously treated with standard-size-particle ICS alone; or 2) small-particle-size BDP/formoterol combination (F) (Chiesi Farmaceutici Spa) for patients previously treated with a standard-particle-size ICS/LABA combination [7-10].

The initial and final visits comprised the following: 1) physical examination; 2) asthma control test (ACT) assessment; and 3) lung function and nitrogen single-breath testing and impulse oscillometry (Vmax and Jaeger Master Screen-IOS; Carefusion Technologies, San Diego, CA, USA). Smoking was not permitted $1 \mathrm{~h}$ prior to testing. This study was approved by the local ethics committee, and patients provided signed informed consent before enrolment.

The primary study outcome was the phase III slope $\left(\mathrm{dN}_{2}\right)$ during a single-breath nitrogen washout test (SBNT) at baseline. Secondary outcomes included airway resistance at different frequencies (R5, R5-R20) as measured by impulse oscillometry, lung volume, and asthma control. Pre-specified outcomes included changes in the functional and clinical outcomes at the end of the 3-month small-particle-size treatment intervention. As no previous studies specifically investigated $\mathrm{dN}_{2}$ via SBNT in smoking asthmatic patients, we based our sample size calculation on the existing literature regarding small airway impairment in asthmatic patients $[11,12]$. We estimated that 23 patients/group would be sufficient to detect inter-group differences in the $\mathrm{dN}_{2}$ of at least $25 \%$ by SBNT (primary study outcome). At each time point, inter-group differences were calculated using an unpaired t-test or the Mann-Whitney test. Paired t-tests or Wilcoxon matched pair tests, linear regression analysis, and the Pearson test were used when appropriate. p-values of $<0.05$ were considered significant.

50 asthmatic patients (25 current smokers and 25 nonsmokers matched for demographics, baseline lung volumes, and treatment) completed the study (table 1). The primary objective, $\mathrm{dN}_{2}$ measured by SBNT, was significantly higher in smokers, compared with nonsmoking asthmatic patients, indicating increased ventilation heterogeneity in the small airways of smokers with asthma (table 1). Impulse oscillometry

TABLE 1 Summary of the baseline patient characteristics and main study findings.

Characteristics
Nonsmoking asthmatic patients

$\begin{array}{cc}\text { Baseline } & \text { After 3-month } \\ \text { small-particle-size } \\ \text { intervention }\end{array}$

Smoking asthmatic patients

\begin{tabular}{cc}
\hline Baseline & $\begin{array}{c}\text { After 3-month } \\
\text { small-particle-size } \\
\text { intervention }\end{array}$
\end{tabular}

\begin{tabular}{l}
\hline Subjects \\
Sex male/female \\
Age years \\
Duration of the disease years \\
Smoking habit pack-year \\
Standard-size particle beclomethas \\
equivalent dose $\mu$ g daily \\
Patients on inhaled ICS alone \% \\
Patients on ICS/LABA fixed combo \\
Asthma control test score \\
FEV \\
Pre-bronchodilator L \\
Pre-bronchodilator \% predicted \\
Post-bronchodilator \\
Post-bronchodilator \% predicted \\
Residual volume \% predicted \\
Diffusion capacity \% predicted \\
Use of rescue medication ${ }^{\#}$ \\
R5 $\mathrm{kPa} \cdot \mathrm{L}^{-1} \mathrm{~s}^{-1}$ \\
R5-R20 $\mathrm{kPa}^{-1} \mathrm{~L}^{-1} \cdot \mathrm{s}^{-1}$ \\
dN $\mathrm{N}_{2} \% \cdot \mathrm{L}^{-1}$
\end{tabular}

$\begin{array}{cc}25 & \\ 13 / 12 & \\ 36.9 \pm 2.0 & \\ 14.2 \pm 1.8 & \\ 376 \pm 13 & \\ & \\ 24 & \\ 76 & \\ 21.2 \pm 0.4(18-25) & 21.9 \pm 0.4(19-25) \\ & \\ 2.76 \pm 0.14 & 2.71 \pm 0.18 \\ 84 \pm 2.3 & 82.4 \pm 2.6 \\ 3.18 \pm 0.18 & 3.16 \pm 0.14 \\ 96 \pm 2.5 & 95.3 \pm 2.1 \\ 103.2 \pm 3.5 & 101.2 \pm 2.9 \\ 88.8 \pm 1.2 & 88.6 \pm 1.1 \\ 0.8 \pm 0.2 & 0.9 \pm 0.3 \\ 0.40(0.28-0.84) & 0.37(0.21-1.00) \\ 0.02(0.002-0.13) & 0.03(0.002-0.19) \\ 1.70(1.08-3.70) & 1.9(0.9-4.25)\end{array}$

25

$14 / 11$

$39.7 \pm 2.0$

$12.3 \pm 1.6$

$14.7 \pm 0.8$

$385 \pm 11$

20

80

$20.2 \pm 0.5(16-25)$

$2.83 \pm 0.14$

$82 \pm 2.6$

$3.22 \pm 0.15$

$93 \pm 2.9$

$102.4 \pm 3.7$

$88.4 \pm 1.1$

$0.9 \pm 0.3$

$0.43(0.29-1.01)$

$0.09(0.002-0.25) *$

$3.30(1.00-8.40)^{*}$
$21.9 \pm 0.4(19-25)$

$2.80 \pm 0.16$

$81.1 \pm 2.8$

$3.25 \pm 0.16$

$93.8 \pm 3.0$

$100 \pm 2.7$

$89.3 \pm 1.3$

$0.6 \pm 0.2$

$0.39(0.20-1.02)$

$0.03(0.004-0.18)^{* *}$

$2.5(0.9-6.8)^{* *}$

Data are presented as $\mathrm{n}$, mean \pm SEM, mean \pm SEM (range) or median (range), unless otherwise stated. ICS: inhaled corticosteroids; LABA: long-acting $\beta_{2}$-agonists; $F E V 1$ : forced expiratory volume in $1 \mathrm{~s}$; R5: total airway resistance at $5 \mathrm{~Hz}$; R5-R20: difference between resistance at $5 \mathrm{~Hz}$ and resistance at $20 \mathrm{~Hz}_{\mathrm{dN}}$ : slope of phase III during a single breath nitrogen washout test. ${ }^{\#}$ : albuterol measured in puffs per week in the month prior to the study visit. *: $p<0.05$ versus nonsmoking asthmatic patients at baseline; ${ }^{* *}: p<0.01$ versus baseline. 
testing was performed as an additional sensitivity assessment. The R5-R20, which reflects the contributions of small airways to airway resistance, but not R5 (total airway resistance), was significantly higher in smokers, compared to nonsmoking asthmatic patients (table 1), in accordance with previous descriptive reports [13] that envisioned an increasingly impaired small airway function in smoking relative to nonsmoking asthmatic patients. However, pathologic events that lead to increased ventilation heterogeneity and peripheral airway resistance in smoking asthmatics are largely unknown. In a recent study, computed tomography revealed a reduced lumen area in the smaller airways of smoking asthmatic patients, suggesting that peripheral airway narrowing might contribute to small airway dysfunction [14].

In this study, no inter-group differences were observed in the baseline ACT score or the use of rescue medication in the month before the study (table 1). However, a higher percentage of smoking asthmatics reported an ACT score $\leqslant 19$, compared to nonsmokers (32 versus $20 \%$ for smoking versus nonsmoking asthmatics, respectively; $\mathrm{p}=0.09$ ). We further identified an inverse correlation between the level of asthma control and the magnitude of the $\mathrm{dN}_{2}$ in smoking asthmatic patients $(\mathrm{p}<0.05, \mathrm{r}=-0.47)$, but not in nonsmoking asthmatic patients, indicating the impact of small airway abnormalities associated with smoking on relevant clinical outcomes, such as asthma control.

The 3-month small-particle-size inhaled formulation treatment intervention led to significantly reduced $\mathrm{dN}_{2}$ and R5-R20 values in smoking asthmatic patients (table 1). These reductions were accompanied by a significant improvement in the ACT scores among subjects with a baseline ACT score $\leqslant 19$ (mean \pm SEM of improvement $2.5 \pm 0.26$ units from the baseline, $\mathrm{p}<0.05$ ). No significant changes in small airway measurements were observed in nonsmoking asthmatic patients although, notably, the baseline $\mathrm{dN}_{2}$ and $\mathrm{R} 5-\mathrm{R} 20$ values of nonsmoking asthmatic patients were near the lower limit of normality. Taken together, these data indicate that small-particle-size treatments could effectively reverse smoking-related small airway abnormalities in smoking asthmatics. Of course, as in all clinical trials, we cannot exclude a study participation effect.

An ancillary finding of this study was the significantly greater improvement in $\mathrm{dN}_{2}$ following treatment with the $\mathrm{BDP} / \mathrm{F}$ combination (changes in $\mathrm{dN}_{2}-1.43 \pm 0.23 \% \cdot \mathrm{L}^{-1}$ ) versus $\mathrm{BDP}$ alone (changes in $\mathrm{dN}_{2}$ $\left.-0.54 \pm 0.21 \% \cdot \mathrm{L}^{-1}\right), \mathrm{p}<0.05$ in smoking asthmatics receiving small-particle-size treatment. However, given the small number of patients treated with BDP alone (table 1) this analysis can be considered only exploratory.

This study was the first to identify small airway dysfunction in smoking asthmatics as a primary objective. Although smoking cessation unquestionably remains the primary therapeutic option for these patients, this study has provided a mechanism to explain the efficacy of small-particle-size treatment in smoking asthmatic patients $[5,15]$.

0 @ERSpublications

Cigarette smoking enhances small airways impairment in asthmatic patients http://ow.ly/WWUky

Marco Contoli ${ }^{1}$, Federico Bellini ${ }^{1}$, Luca Morandi ${ }^{1}$, Giacomo Forini ${ }^{1}$, Stefano Bianchi ${ }^{2}$, Giulia Gnesini ${ }^{1}$, Brunilda Marku ${ }^{1}$, Klaus F. Rabe ${ }^{3,4}$ and Alberto Papi ${ }^{1}$

${ }^{1}$ Research Centre on Asthma and COPD, Dept of Medical Sciences, University of Ferrara, Ferrara, Italy. ${ }^{2}$ Dept of Pharmacy, University Hospital of Ferrara, Ferrara, Italy. ${ }^{3}$ LungClinic Grosshansdorf, Member of the German Center for Lung Research, Grosshansdorf, Germany. ${ }^{4}$ Dept of Medicine, Christian Albrechts University Kiel, Member of the German Center for Lung Research, Kiel, Germany.

Correspondence: Alberto Papi, Research Centre on Asthma and COPD, Department of Medical Sciences, University of Ferrara, Via Savonarola 9, 44121 Ferrara, Italy. E-mail: ppa@unife.it

Received: Oct 152015 | Accepted after revision: Dec 062015 | First published online: Feb 122016

Clinical trial: This study is registered at www.clinicaltrials.gov with identifier number NCT01620099.

Support statement: This work was supported by an unrestricted grant from Chiesi Farmaceutici SpA, Prot. nr. 1214 registered at Department of Medical Sciences, University of Ferrara, Italy.

Conflict of interest: Disclosures can be found alongside the online version of this article at erj.ersjournals.com

Acknowledgements: We thank Elisa Veratelli (University of Ferrara, Ferrara, Italy) for assistance with the manuscript preparation.

\section{References}

1 Contoli M, Kraft M, Hamid Q, et al. Do small airway abnormalities characterize asthma phenotypes? In search of proof. Clin Exp Allergy 2012; 42: 1150-1160.

2 Thomson NC, Chaudhuri R. Asthma in smokers: challenges and opportunities. Curr Opin Pulm Med 2009; 15: 39-45.

3 Labiris NR, Dolovich MB. Pulmonary drug delivery. Part I: physiological factors affecting therapeutic effectiveness of aerosolized medications. Br J Clin Pharmacol 2003; 56: 588-599. 
4 Gentile DA, Skoner DP. New asthma drugs: small molecule inhaled corticosteroids. Curr Opin Pharmacol 2010; 10: $260-265$

5 Roche N, Postma DS, Colice G, et al. Differential effects of inhaled corticosteroids in smokers/ex-smokers and nonsmokers with asthma. Am J Respir Crit Care Med 2015; 191: 960-964.

6 Global Initiative for Asthma. Global Strategy for Asthma Management and Prevention: updated 2015. Geneva Global Initiative for Asthma, 2015. Available from: http://www.ginasthma.org/local/uploads/files/GINA_Report_ 2015_Aug11.pdf

7 Papi A, Paggiaro PL, Nicolini G, et al. Beclomethasone/formoterol versus budesonide/formoterol combination therapy in asthma. Eur Respir J 2007; 29: 682-689.

8 Papi A, Paggiaro P, Nicolini G, et al. Beclomethasone/formoterol vs fluticasone/salmeterol inhaled combination in moderate to severe asthma. Allergy 2007; 62: 1182-1188.

9 Busse WW, Brazinsky S, Jacobson K, et al. Efficacy response of inhaled beclomethasone dipropionate in asthma is proportional to dose and is improved by formulation with a new propellant. J Allergy Clin Immunol 1999; 104: 1215-1222.

10 Huchon G, Magnussen H, Chuchalin A, et al. Lung function and asthma control with beclomethasone and formoterol in a single inhaler. Resp Med 2009; 103: 41-49.

11 Battaglia S, den Hertog H, Timmers M, et al. Small airways function and molecular markers in exhaled air in mild asthma. Thorax 2005; 60: 639-644.

12 van Veen IH, Sterk PJ, Schot R, et al. Alveolar nitric oxide versus measures of peripheral airway dysfunction in severe asthma. Eur Respir J 2006; 27: 951-956.

13 Clearie KL. McKinlay L, Williamson PA, et al. Fluticasone/salmeterol combination confers benefits in people with asthma who smoke. Chest 2012; 141: 330-338.

14 Thomson NC, Chaudhuri R, Spears M, et al. Poor symptom control is associated with reduced CT scan segmental airway lumen area in smokers with asthma. Chest 2015; 147: 735-744.

15 Brusselle G, Peché R, Van den Brande P, et al. Real-life effectiveness of extrafine beclometasone dipropionate/ formoterol in adults with persistent asthma according to smoking status. Resp Med 2012; 106: 811-819.

\section{Decreased breath excretion of redox active iron in COPD: a protective failure?}

To the Editor:

Recent observational and genetic studies have highlighted a potential role for disrupted iron homeostasis in stable and exacerbating chronic obstructive pulmonary disease (COPD). Thus iron deficiency that is not limited to anaemia [1] and single nucleotide polymorphism in the gene encoding iron regulatory protein-2 [2], a key regulatory factor involved in cellular iron turnover and control, have been identified in this population. Disrupted systemic iron homeostasis is likely to limit iron availability for metabolic purposes due to overriding effects on tissue storage rather than on mobilisation and limitation of uptake from the gut. A specific pro-oxidant pool of iron (free or loosely bound ions, which are redox active/catalytic for damaging oxidant production) is measurable in exhaled breath condensate (EBC) [3]. While iron is essential for life, particularly for aerobes, poor or altered iron handling results in adverse effects related to oxidant production, microbial virulence, altered redox signalling events and altered cellular fate, including remodelling. This study was therefore undertaken to gain insight into iron handling in lungs and airways, and the extent to which these processes may be altered in COPD. Studies were undertaken to measure this specific iron pool by the bleomycin method [4] utilising EBC samples collected as previously described [3] and obtained from normal healthy individuals, healthy smokers and patients with COPD (current and ex-smokers). Serum levels of hepcidin and interleukin (IL)-6, known regulators of iron homeostasis, were also measured.

Comparisons of redox active iron content in EBC from age-matched healthy controls, healthy smokers and subjects with COPD, grouped as current and ex-smokers, revealed significant differences between groups (figure 1a). Healthy smokers returned greatly elevated levels of redox active iron in EBC as expected, given that cigarette smoke is known to contain iron and other metallic components [5]. However, no such response was evident in COPD EBC regardless of a current or past smoking history, with values in both groups of the same order as found in samples from healthy controls. There was no significant effect of COPD severity on exhaled redox active iron although a trend was evident (data not shown). In a previous study, which examined redox active iron content in EBC in a variety of subjects (asthma, cardiac surgery and smokers) and settings, the results obtained gave reason to speculate that the presence of this specific and reactive iron pool in EBC may represent an excretory function that may become compromised by 